TITLE:

\title{
True atomic resolution in liquid by frequency-modulation atomic force microscopy
}

$\operatorname{AUTHOR}(\mathrm{S})$ :

Fukuma, T; Kobayashi, K; Matsushige, K; Yamada, H

CITATION:

Fukuma, T ... [et al]. True atomic resolution in liquid by frequencymodulation atomic force microscopy. APPLIED PHYSICS LETTERS 2005, 87(3): 034101.

\section{ISSUE DATE:}

2005-07-18

URL:

http://hdl.handle.net/2433/39675

\section{RIGHT:}

Copyright 2005 American Institute of Physics. This article may be downloaded for personal use only. Any other use requires prior permission of the author and the American Institute of Physics. 


\title{
True atomic resolution in liquid by frequency-modulation atomic force microscopy
}

\author{
Takeshi Fukuma \\ Department of Electronic Science and Engineering, Kyoto University, \\ Katsura, Nishikyo, Kyoto 615-8510, Japan \\ Kei Kobayashi \\ International Innovation Center, Kyoto University, Yoshida-Honmachi, Sakyo, Kyoto 606-8501, Japan \\ Kazumi Matsushige and Hirofumi Yamada ${ }^{\text {a) }}$ \\ Department of Electronic Science and Engineering, Kyoto University, \\ Katsura, Nishikyo, Kyoto 615-8510, Japan
}

(Received 29 March 2005; accepted 20 June 2005; published online 12 July 2005)

True atomic resolution of frequency-modulation atomic force microscopy in liquid is demonstrated. Hexagonal lattice of a cleaved (001) surface of muscovite mica is resolved in water. Nonperiodic structures such as defects and adsorbates are simultaneously imaged with the atomic-scale features of mica surface. The use of small oscillation amplitude $(0.16-0.33 \mathrm{~nm})$ of a force sensing cantilever allows us to obtain vertical and lateral resolutions of 2-6 and $300 \mathrm{pm}$, respectively, even with a low $Q$ factor in water $(Q=20-30)$. (C) 2005 American Institute of Physics. [DOI: 10.1063/1.1999856]

Frequency-modulation atomic force microscopy $(\text { FM-AFM })^{1}$ has a distinctive advantage over other scanning probe techniques such as scanning tunneling microscopy ${ }^{2}$ and contact-mode atomic force microscopy $(\mathrm{CM}-\mathrm{AFM}):^{3}$ the imaging ability of insulating surfaces with true atomic (i.e., subnanometer-scale) resolution. ${ }^{4}$ This ability is essential for the applications in organic molecular science and nanobiology where molecular-scale investigations on nonconductive and soft organic materials are required. Although a large number of subnanometer-scale FM-AFM images have been presented so far, ${ }^{4-9}$ such high-resolution imaging has been successful only in ultrahigh vacuum (UHV) environment. This limitation has prevented a wide range of its applications in air and liquid. In particular, high-resolution imaging in liquid is indispensable for investigating biological samples in their physiological environments.

The major difficulty in high-resolution FM-AFM imaging in liquid is the low force sensitivity due to the low $Q$ factor of the cantilever resonance. ${ }^{10,11}$ Recently, the authors have overcome the difficulty and succeeded in obtaining true molecular resolution in air ${ }^{12}$ and liquid ${ }^{13}$ with a newly developed multienvironment FM-AFM. ${ }^{14}$ We used a home-built cantilever deflection sensor ${ }^{14}$ having a deflection noise density of $17 \mathrm{fm} / \sqrt{\mathrm{Hz}}$ for obtaining a maximum force sensitivity limited by the cantilever thermal Brownian motion. The remarkable noise characteristic of the deflection sensor also allows us to maintain the cantilever oscillation amplitude $(A)$ at as small as $0.2-0.3 \mathrm{~nm}$ in liquid. This small amplitude operation is extremely effective for enhancing the sensitivity to the short-range interaction forces ${ }^{15}$ and thereby obtaining a high spatial resolution. ${ }^{16}$ In this letter, we present the first result showing true atomic resolution of FM-AFM in liquid. A cleaved (001) surface of muscovite mica is imaged in water using the above mentioned technique and instruments.

Figure 1(a) shows $a$-axis projection of the crystal structure of muscovite mica $\left[\mathrm{KAl}_{2}\left(\mathrm{Si}_{3} \mathrm{Al}\right) \mathrm{O}_{10}(\mathrm{OH})_{2}\right]$. The crystal

\footnotetext{
${ }^{a)}$ Electronic mail: h-yamada@kuee.kyoto-u.ac.jp
}

consists of complicated aluminosilicate layers separated by $\mathrm{K}^{+}$ions. In the bulk crystal, one-fourth of $\mathrm{Si}^{4+}$ ions are replaced with $\mathrm{Al}^{3+}$ ions so that the layer is negatively charged. This negative charge is compensated by the $\mathrm{K}^{+}$ion layer which electrostatically bounds the adjacent two aluminosilicate layers. The crystal is easily cleaved at the $\mathrm{K}^{+}$ion layer, presenting an atomically flat surface as shown in Fig. 1(b). The surface is composed of $\mathrm{Si}$ (partially $\mathrm{Al}$ ) and $\mathrm{O}$ atoms forming an array of hexagons with a unit cell length of 0.52 nm.

Figure 2 shows an FM-AFM image of the cleaved (001) surface of muscovite mica taken in water. Atomic-scale features having a period of $0.52 \mathrm{~nm}$ are clearly imaged with large-scale corrugations and some adsorbates. Although a number of atomic-scale images of mica have been presented by CM-AFM, ${ }^{17,18}$ simultaneous imaging of atomic-scale features with such surface corrugations and adsorbates have not been successful. This is because the average load force is relatively large and the tip motion produces a high lateral force in CM-AFM. In FM-AFM, the vertical oscillation of the cantilever reduces both vertical load and lateral frictional force, which allows us to image atomic-sale details even (a) $a$-axis projection

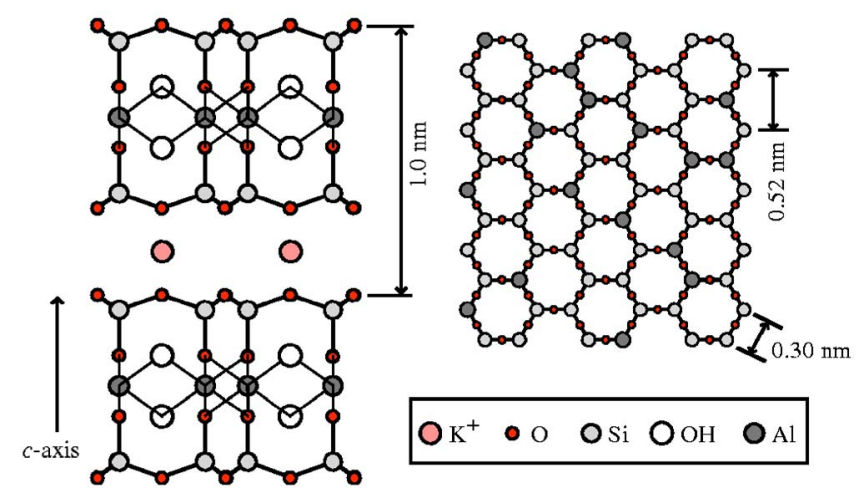

FIG. 1. (Color online) The crystal structure of muscovite mica; (a) $a$-axis projection; (b) cleaved surface ( $\mathrm{K}^{+}$ions are not shown). 


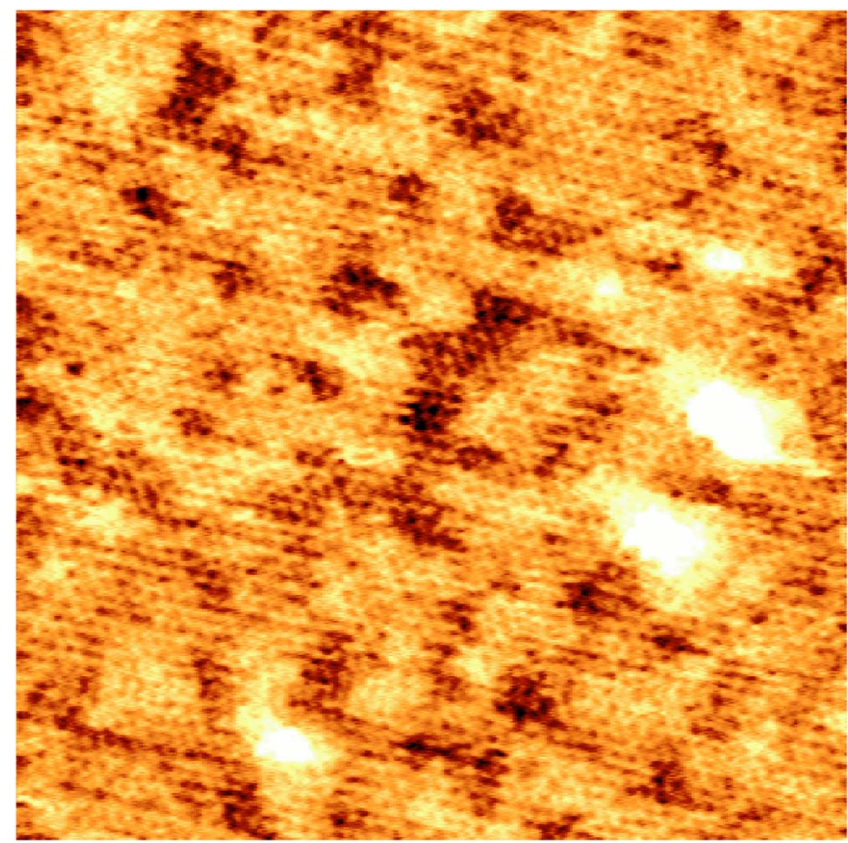

FIG. 2. (Color online) FM-AFM image of the cleaved (001) surface of muscovite mica taken in water $(30 \mathrm{~nm} \times 30 \mathrm{~nm}, \Delta f=+364 \mathrm{~Hz}, A$ $=0.33 \mathrm{~nm}$, scanning speed: $561 \mathrm{~nm} / \mathrm{s}$ ). The tip-sample distance regulation was made in constant frequency shift mode. The cantilever used was an $n-\mathrm{Si}$ cantilever (Nanosensors: $\mathrm{NCVH}$ ) with a spring constant of $37 \mathrm{~N} / \mathrm{m}$ and a resonance frequency of $176 \mathrm{kHz}$ in water. The $Q$ factor measured in water was 23 .

with randomly distributed adsorbates weakly bound to the surface. This capability is essential for the applications in nanobiology where subnanometer-scale investigations on weakly bound biological materials are required.

The FM-AFM images of mica taken in water show two different contrast patterns depending on the frequency shifts $(\Delta f)$ used for the tip-sample distance regulation: honeycomb-like pattern [Fig. 3(a)] and dot-like pattern [Fig. 3(b)]. The honeycomb-like pattern consists of a number of hexagons repeating with a period of $0.52 \mathrm{~nm}$. This contrast pattern is found in the FM-AFM images taken with relatively small frequency shifts (less than $+200 \mathrm{~Hz}$ when $A$ $=0.20 \mathrm{~nm}$ ), namely relatively weak tip-sample interaction force. This contrast pattern agrees well with the atomic-scale structure of mica surface shown in Fig. 1(b). The FM-AFM image shown in Fig. 3(c) presents more detailed structure of the honeycomb-like pattern. The image shows some bright spots on the hexagons. The structural model shown in Fig. 1(b) suggests that the bright spots found in the image are located on the $\mathrm{Si}$ atom sites in the hexagons. These bright spots may correspond to the $\mathrm{Al}^{3+}$ ions because $\mathrm{Al}^{3+}$ ions are probably imaged as relatively large protrusions due to their negative charges offered from the surrounding $\mathrm{O}^{2-}$ ions. The distance between the adjacent bright spots is about $300 \mathrm{pm}$, showing a good agreement with the distance between the two adjacent Si atom sites [Fig. 1(b)]. Such nonperiodic atomicscale contrasts in FM-AFM images demonstrate that true atomic-resolution imaging by FM-AFM is possible even with a $Q$ factor of as low as 30 in water.

The FM-AFM images taken with a relatively large frequency shifts (larger than $+200 \mathrm{~Hz}$ when $A=0.20 \mathrm{~nm}$ ), namely relatively large tip-sample interaction force, show the dotlike pattern as shown in Fig. 3(b). The dotlike pattern consists of hexagonally arranged bright dots separated by Downloaded 30 May 2007 to 130.54.110.22. Redistribution subject
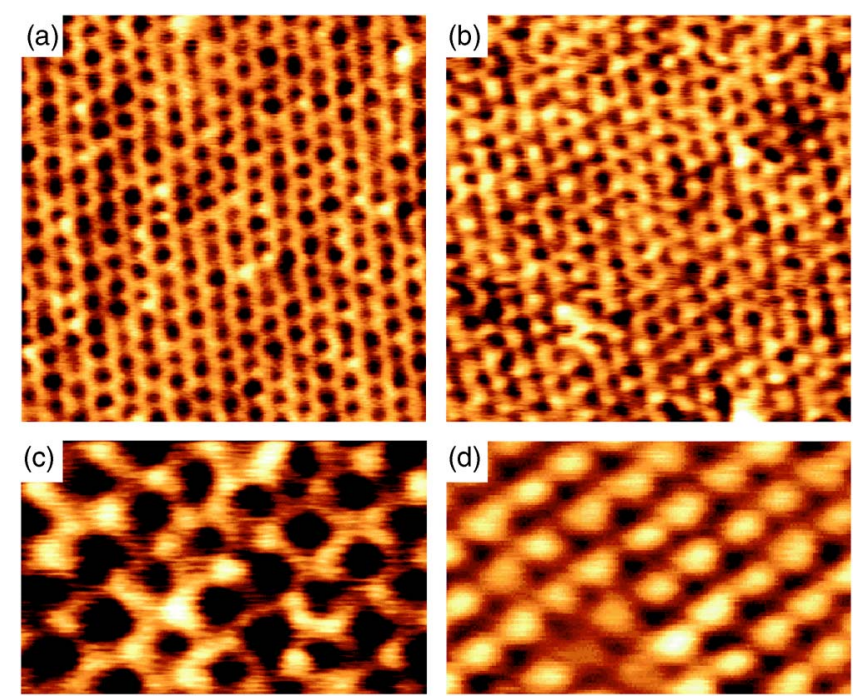

FIG. 3. (Color online) FM-AFM images of the cleaved (001) surface of muscovite mica taken in water. (a) $8 \mathrm{~nm} \times 8 \mathrm{~nm}, \Delta f=+54 \mathrm{~Hz}, A$ $=0.24 \mathrm{~nm}$, scanning speed: $671 \mathrm{~nm} / \mathrm{s}$; (b) $8 \mathrm{~nm} \times 8 \mathrm{~nm}, \Delta f=+240 \mathrm{~Hz}, A$ $=0.20 \mathrm{~nm}$, scanning speed: $1120 \mathrm{~nm} / \mathrm{s}$; (c) $4 \mathrm{~nm} \times 2.5 \mathrm{~nm}, \Delta f=+157 \mathrm{~Hz}$, $A=0.16 \mathrm{~nm}$, scanning speed: $934 \mathrm{~nm} / \mathrm{s}$; (d) $4 \mathrm{~nm} \times 2.5 \mathrm{~nm}, \Delta f=+682 \mathrm{~Hz}$, $A=0.20 \mathrm{~nm}$, scanning speed: $671 \mathrm{~nm} / \mathrm{s}$. The images were taken in constant height mode. The cantilever used was an $n$-Si cantilever (Nanosensors: $\mathrm{NCH}$ ) with a spring constant of $42 \mathrm{~N} / \mathrm{m}$ and a resonance frequency of 136 $\mathrm{kHz}$ in water. The $Q$ factor measured in water was 30 .

$0.52 \mathrm{~nm}$. Under this condition, some of the bright dots show asymmetric contrast in the fast scanning direction [Fig. 3(d)], indicating the influence of the tip-sample frictional interaction. The dot-like pattern is similar to those of "lattice images" obtained by CM-AFM ${ }^{17,18}$ where only periodic structures are imaged through the frictional interaction averaged over a relatively large tip-sample contact area. However, FM-AFM images obtained in this experiment also show subnanometer-scale structural defects as shown in Fig. 3(d). This clear difference from the lattice images reveals that the image was formed through the frictional interactions acting in the subnanometer-scale tip-sample contact area.

The precise control of the vertical tip position near the tip-sample contact point makes it possible to probe a wide range of interactions from atom-to-atom interactions for true atomic resolution [Fig. 3(c)] to frictional interactions in the subnanometer-scale contact area [Fig. 3(d)]. This ability also allows us to obtain a high spatial resolution with sufficiently small load forces. We have quantitatively investigated the vertical resolution of FM-AFM in water from the frequency shift-distance curve measured on mica (Fig. 4). The curve shows a force oscillation with a period of about $0.16 \mathrm{~nm}$ probably due to the layering of the water molecules confined in the tip-sample separation. ${ }^{19,20}$ True atomic resolution was stably obtained with frequency shifts in the range from $+250 \mathrm{~Hz}$ to $+500 \mathrm{~Hz}$ (gray shadow in Fig. 4). From the curve shown in Fig. 4, the frequency shift gradient in this frequency shift range is $|\partial(\Delta f) / \partial z|=3-9 \times 10^{13} \mathrm{~Hz} / \mathrm{m}$. On the other hand, the frequency noise density measured with a fast Fourier transform analyzer shows a nearly constant value of $0.58 \mathrm{~Hz} / \sqrt{\mathrm{Hz}}$ at frequencies less than the FM bandwidth of $1 \mathrm{kHz}$. Thus, the frequency noise is $\delta f=18.4 \mathrm{~Hz}$ with an FM bandwidth of $1 \mathrm{kHz}$. Accordingly, the vertical resolution of FM-AFM is $\delta z=|\delta f /(\partial(\Delta f) / \partial z)|=2-6 \mathrm{pm}$ in this frequency shift range. Since the vertical corrugation of the atomic surface is typically $20-100 \mathrm{pm},{ }^{21}$ vertical resolution to AIP license or copyright, see http://apl.aip.org/apl/copyright.jsp 


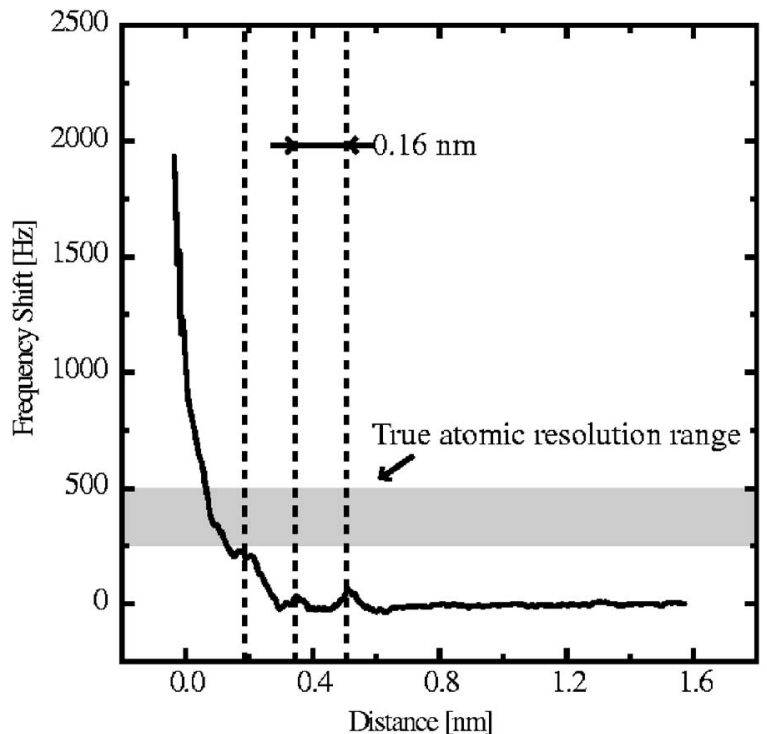

FIG. 4. Frequency shift-distance curve measured on a cleaved (001) surface of muscovite mica in water $\left(A=0.33 \mathrm{~nm}, Q=19, f_{0}=155 \mathrm{kHz}, k=42 \mathrm{~N} / \mathrm{m}\right)$. The curve was taken with a tip velocity of $0.7 \mathrm{~nm} / \mathrm{s}$ and approximately 1000 data points. The tip-sample contact point is not identified.

of $2-6 \mathrm{pm}$ is sufficiently small to achieve true atomic resolution. The result indicates that the signal enhancement owing to the small-amplitude operation overcomes the large frequency noise due to the low $Q$ factor in water.

The success of true atomic resolution imaging by FMAFM in water opens a wide variety of new applications that cannot be performed by CM-AFM. The small tip-sample friction force in FM-AFM allows us to investigate atomicscale phenomena taking place at the liquid/solid interface through weak interaction forces. For example, molecularscale investigations on the water layers on hydrophilic surfaces such as mica is one of the promising future subjects.

The support of a Grant-in-Aid and a Leading Project on Nanotechnology and Materials from the Ministry of Education, Science, Sports and Culture of Japan, and 21st Century
Center of Excellence Program, Kyoto University are gratefully acknowledged.

${ }^{1}$ T. R. Albrecht, P. Grütter, D. Horne, and D. Rugar, J. Appl. Phys. 69, 668 (1991).

${ }^{2}$ G. Binnig, H. Rohrer, C. Gerber, and E. Weibel, Phys. Rev. Lett. 49, 57 (1982).

${ }^{3}$ G. Binnig, C. F. Quate, and C. Gerber, Phys. Rev. Lett. 56, 930 (1986).

${ }^{4}$ M. Bammerlin, R. Lüthi, E. Meyer, A. Baratoff, J. Lü, M. Guggisberg, C. Gerber, L. Howald, and H.-J. Güntherodt, Probe Microsc. 1, 3 (1997).

${ }^{5}$ F. J. Giessibl, Science 267, 68 (1995).

${ }^{6}$ S. Kitamura and M. Iwatsuki, Jpn. J. Appl. Phys., Part 2 34, L1086 (1995).

${ }^{7}$ K. Fukui, H. Onishi, and Y. Iwasawa, Chem. Phys. Lett. 280, 296 (1997).

${ }^{8}$ S. Orisaka, T. Minobe, T. Uchihashi, Y. Sugawara, and S. Morita, Appl. Surf. Sci. 140, 243 (1999).

${ }^{9}$ C. Loppacher, M. Bammerlin, M. Guggisberg, F. Battiston, R. Bennewitz, S. Rast, A. Baratoff, E. Meyer, and H.-J. Güntherodt, Appl. Surf. Sci. 140, 287 (1999).

${ }^{10}$ P. K. Hansma, J. P. Cleveland, M. Radmacher, D. A. Walters, P. E. Hilner, M. Bezanilla, M. Fritz, D. Vie, and H. G. Hansma, Appl. Phys. Lett. 64, 1738 (1994).

${ }^{11}$ C. A. J. Putman, K. O. V. der Werf, G. D. Grooth, and N. F. V. Hulst, Appl. Phys. Lett. 64, 2454 (1994).

${ }^{12}$ T. Fukuma, T. Ichii, K. Kobayashi, H. Yamada, and K. Matsushige, Appl. Phys. Lett. 86, 034103 (2005).

${ }^{13}$ T. Fukuma, K. Kobayashi, K. Matsushige, and H. Yamada, Appl. Phys. Lett. 86, 193108 (2005).

${ }^{14}$ T. Fukuma, M. Kimura, K. Kobayashi, K. Matsushige, and H. Yamada, Rev. Sci. Instrum. 76, 053704 (2005).

${ }^{15}$ F. J. Giessibl, H. Bielefeldt, S. Hembacher, and J. Mannhart, Appl. Surf. Sci. 140, 352 (1999).

${ }^{16}$ F. J. Giessibl, S. Hembacher, H. Bielefeldt, and J. Mannhart, Science 289, 422 (2000).

${ }^{17}$ R. Erlandsson, G. Hadziioannou, C. M. Mate, G. M. McClelland, and S. Chiang, J. Chem. Phys. 89, 5190 (1988).

${ }^{18}$ B. Drake, C. B. Prater, A. L. Weisenhorn, S. A. Gould, T. R. Albrecht, C. F. Quate, D. S. Cannell, H. G. Hansma, and P. K. Hansma, Science 243, 1586 (1989).

${ }^{19}$ J. P. Cleveland, T. E. Schäffer, and P. K. Hansma, Phys. Rev. B 52, R8692 (1995).

${ }^{20}$ S. P. Jarvis, T. Uchihashi, T. Ishida, and H. Tokumoto, J. Phys. Chem. 104, 6091 (2000).

${ }^{21}$ Noncontact Atomic Force Microscopy Nanoscience and Technology, edited by S. Morita, R. Wiesendanger, and E. Meyer (Springer, Berlin, 2002). 